\title{
Giving out dangerous signals
}

Microglia mediate potent innate immune responses to both infectious and traumatic challenges. However, they are not always neuroprotective, as upon activation they can accelerate neurodegeneration. The precise mechanism by which this happens is unclear. Now, Lehnardt et al. report that release of the heat-shock protein HSP60 from injured neurons endogenously activates microglia and brings about neuronal demise.

The authors found that neurons isolated from mice bearing a

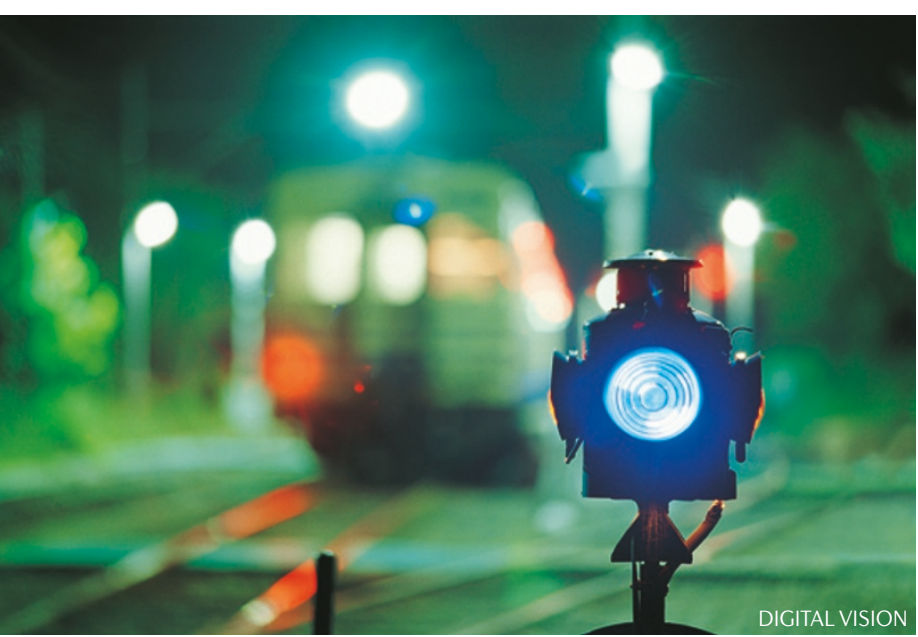

mutation that inactivates toll-like receptor 4 (TLR4) signalling, which is known to mediate the innate immune response, were less vulnerable to cell death caused by the isolation procedure. This led them to suspect that release of an endogenous TLR4 ligand from injured neurons contributes to this cell death.

HSP60 has previously been reported to bind to TLR4 and exert a pro-inflammatory effect that is dependent on functional TLR4 signalling. Here, the authors showed that neurons release HSP60 when undergoing apoptosis or necrosis, and that both HSP60-containing supernatant from ailing neuronal cultures and recombinant HSP60 had a potent neurotoxic effect on mixed neuronal cultures (containing microglia) but not on purified neuronal cultures. Importantly, this neurotoxic effect was not observed in mixed cultures from Tlr4 mutants or when HSP60 was depleted from the supernatant (by small interfering RNA or with a monoclonal antibody), confirming that the effect was due to the action of HSP60 on microglial TLR4.
Activated TLRs are known to recruit the adaptor protein $\mathrm{MyD} 88$ and induce the production and release of neurotoxic nitric oxide (NO). Here the authors showed that the effects of HSP60 on cell death were abolished in mixed neuronal cultures derived from Myd88 $8^{-/-}$mice or upon inhibition of NO production in wild-type cultures. These findings indicate that the effects of HSP60 on neuron loss are also dependent on MyD88 and the release of NO from microglia.

There is mounting evidence that reducing inflammation in the CNS by preventing microglia activation could have clinical benefits for patients suffering from neurodegenerative diseases or traumatic injury. This paper sheds light on a pathway that could be common to many types of neuronal injury and that links the activation of the immune system with neurodegeneration.

Monica Hoyos Flight

ORIGINAL RESEARCH PAPER Lehnardt, S. et al. $A$ vicious cycle involving release of heat shock protein 60 from injured cells and activation of toll-like receptor 4 mediates neurodegeneration in the CNS. J. Neurosci. 27, 2320-2331 (2008) 